\title{
La verrière de sainte Anne à Saint-Eusèbe d'Auxerre : les donateurs et leurs armoiries révélés
}

Danielle Velde et Françoise Perrot

\section{Q OpenEdition}

1 Journals

Édition électronique

URL : https://journals.openedition.org/cem/14552

DOI : $10.4000 /$ cem. 14552

ISSN : 1954-3093

Éditeur

Centre d'études médiévales Saint-Germain d'Auxerre

Référence électronique

Danielle Velde et Françoise Perrot, «La verrière de sainte Anne à Saint-Eusèbe d'Auxerre : les donateurs et leurs armoiries révélés », Bulletin du centre d'études médiévales d'Auxerre / BUCEMA [En ligne], 20.2 | 2016, mis en ligne le 06 mars 2017, consulté le 22 septembre 2022. URL : http:// journals.openedition.org/cem/14552 ; DOI : https://doi.org/10.4000/cem.14552

Ce document a été généré automatiquement le 22 septembre 2022.

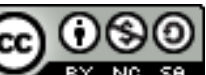

Creative Commons - Attribution - Pas d'Utilisation Commerciale - Partage dans les Mêmes Conditions 4.0 International - CC BY-NC-SA 4.0

https://creativecommons.org/licenses/by-nc-sa/4.0/ 


\title{
La verrière de sainte Anne à Saint- Eusèbe d'Auxerre : les donateurs et leurs armoiries révélés
}

\author{
Danielle Velde et Françoise Perrot
}

1 L'église Saint-Eusèbe, de très ancienne fondation (vII siècle), a connu de nombreuses vicissitudes, sans jamais abandonner son vocable initial. Pour se rapprocher de l'époque qui va nous intéresser, signalons que l'ancien chevet, reconstruit après l'incendie de 1216, s'est effondré en 1523. Le nouveau chœur, mis en chantier en 1530, reçut des vitraux dont la plupart furent à leur tour détruits pendant l'occupation de la ville par les Huguenots en 1567-1568 ${ }^{1}$. Les nouveaux vitraux, offerts par les paroissiens dans le cadre de la restauration, ont été bien maltraités au cours du temps ${ }^{2}$. Finalement une importante reprise eut lieu en 1967,

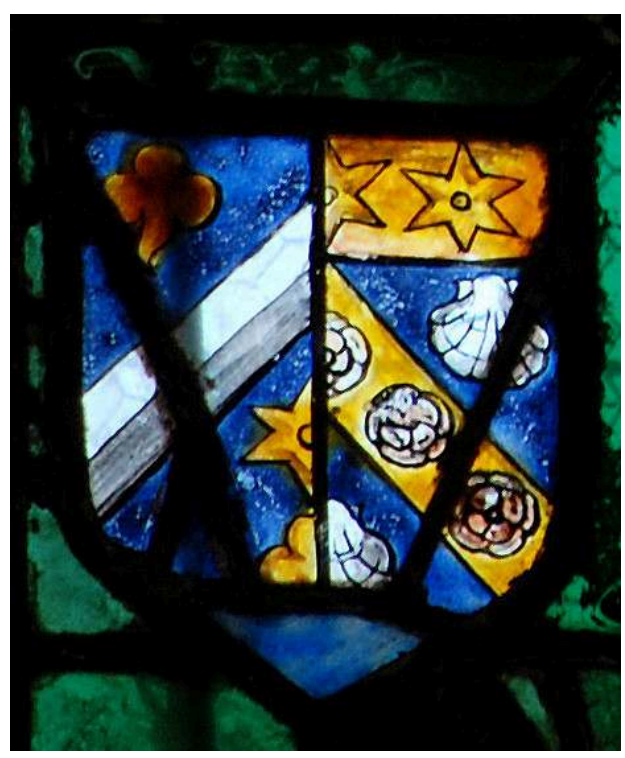
avec des compléments apportés par l'atelier du peintre verrier parisien, Pierre Gaudin. Ceux-ci sont bien visibles dans les verrières du déambulatoire du chœur, qui datent du dernier quart $d u x v I^{e}$ siècle et du premier quart du XvII siècle.

2 C'est le cas de l'avant-dernière baie à deux lancettes au nord-est, fermée par une verrière montrant, dans son état actuel, des épisodes de la vie de sainte Anne et de l'Enfance de la Vierge dans les lancettes et, dans l'ajour central du tympan, la fraction masculine d'une famille de donateurs agenouillés vers la droite: un homme âgé accompagné de quatre adolescents. Les trois écus représentés dans cette verrière 
n'avaient pas été identifiés pour l'article de 2011 et, au vu des documents conservés aux Archives départementales de l'Yonne mentionnant des donations par la famille Debrie, très présente dans la paroisse Saint-Eusèbe, il avait été proposé de lui attribuer cette verrière ${ }^{3}$.

Or, la présente étude propose de rendre à ces donateurs une identité plausible grâce à l'identification de leurs blasons et, au-delà, leur place dans la société d'Auxerre.

4 La première partie $d u$ texte exploitera l'héraldique. Les indications fournies permettront, dans une seconde partie, de nous repérer dans les registres paroissiaux, très lacunaires à Auxerre, et ainsi de rendre une identité plausible aux commanditaires et de cerner la période de leur résidence dans la paroisse Saint-Eusèbe.

\section{L’héraldique}

5 Des trois blasons repérés, le premier blason est simple : D'azur au chevron d'argent accompagné de trois trèfles d'or et d'une molette aussi d'or au nombril de l'écu (amortissement de la lancette gauche). Les deux autres sont partis, avec dans les deux cas, les armes cidessus décrites placées à dextre :

- blason 2: De gueules au sautoir engrelé d'or accompagné de deux lions d'or en chef et en pointe et de deux enfants emmaillotés à dextre et à senestre, posés en fasce (amortissement de la lancette droite) ;

- blason 3 : D'azur au chevron d'or chargé de cinq roses de ? et accompagné de trois coquilles d'argent, au chef de gueules chargé de trois molettes à six raies de gueules (sur le prie-Dieu dans l'oculus central).

Le blason 1 apparaît seul dans l'amortissement de la lancette gauche tandis que, dans l'amortissement de la lancette droite, un blason parti, montre à dextre le blason 1 et à senestre le blason 2 (fig. 1).

Fig. 1 - Église Saint-Eusèbe, partie sommitale de la verrière de sainte Anne, têtes de lancettes avec les blasons au centre d'un chapeau de triomphe

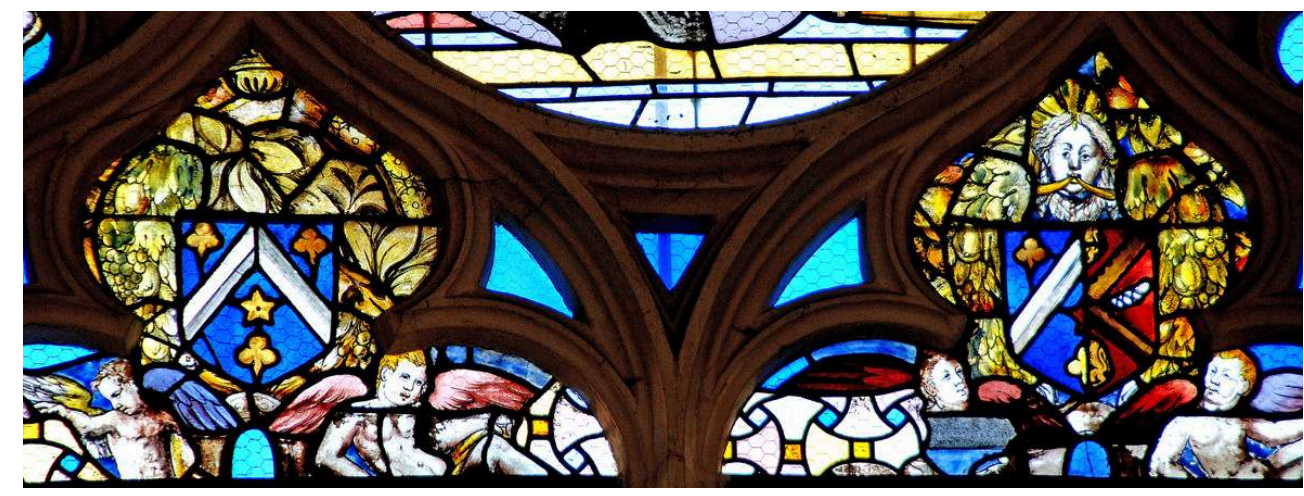

Cl. P. Wahlen

7 L'interprétation simple de cette association est de considérer que le blason 1 représente les armes de l'époux et le second, parti, celui de l'épouse.

8 Le troisième (visible sur la fig. 2), associant à dextre le blason 1 et à sénestre un autre, inconnu, serait celui de l'épouse d'un homme portant le blason 1, associé à un autre qui 
reste à interpréter - et pourrait être celui de la mère du personnage, dont les armoiries sont représentées à gauche sur la figure 1 .

Fig. 2 - Auxerre, église Saint-Eusèbe, verrière de sainte Anne, ajour supérieur du tympan

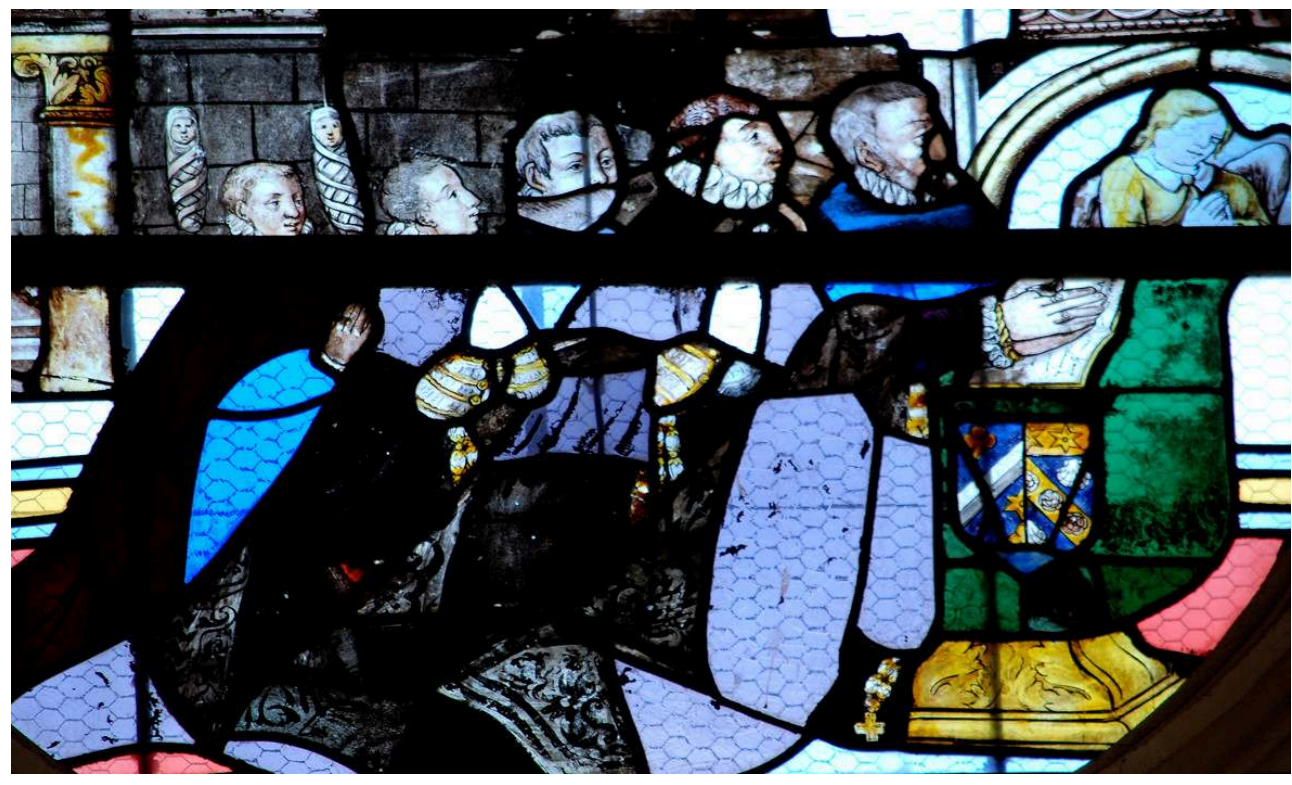

La fraction mâle de la famille, Charles Rousselet accompagné de quatre de ses fils. Un blason visible sur le prie-Dieu. À l'arrière-plan, on distingue deux bébés emmaillotés qui sont des meubles du blason Simonnet, mais pourraient représenter deux fils décédés en bas âge. Cette partie de la verrière est en mauvais état, manques nombreux et multiples bouche-trous.

Cl. P. Wahlen

Le blason 1 ne figure dans aucun répertoire. En revanche le blason 2, vraisemblablement celui de l'épouse du porteur du blason 1, est connu. Il est répertorié, avec des émaux différents, comme appartenant à la famille Simonnet dans deux volumes au moins de l'Armorial général de la France d'Hozier, manuscrits conservés à la Bibliothèque nationale de France ${ }^{4}$ (fig. 3).

Fig. 3 - Reproduction du blason de Claude Simonnet, de Paris

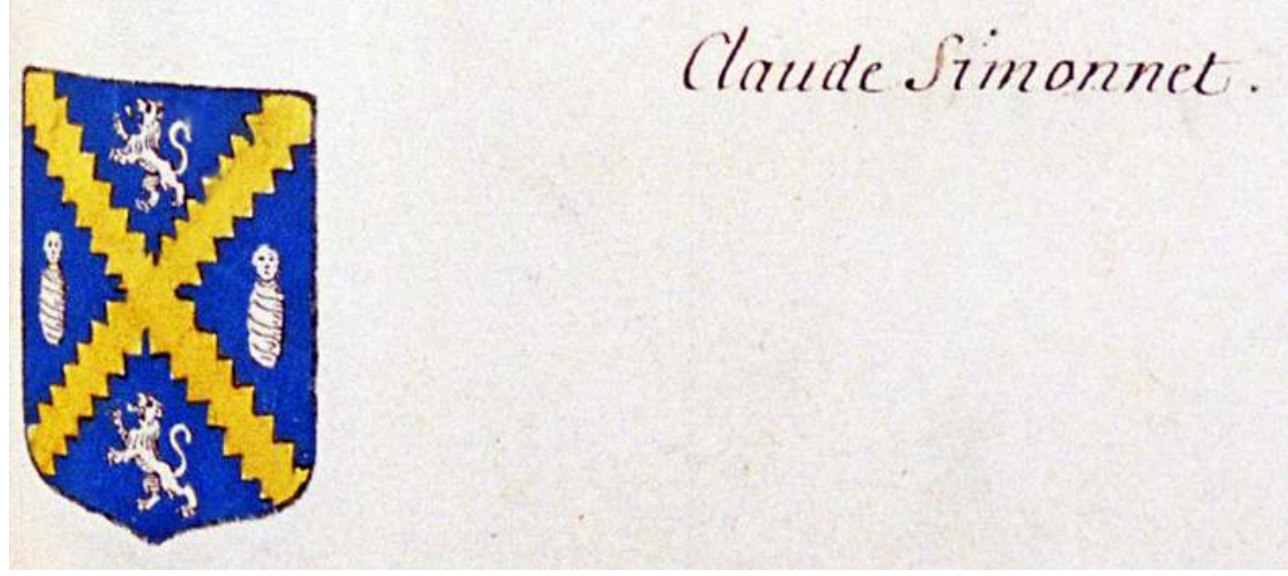

Bibliothèque nationale de France, Manuscrits français, 32251, p. 1497.

L'armorial est postérieur à la période qui nous intéresse et quelques différences sont à noter : à Auxerre, le champ est de gueules et l'enfant emmailloté est posé en fasce, 
tandis que sur les deux blasons figurés dans l'Armorial général le champ est de sable ou d'azur et l'enfant emmailloté posé en pal. Mais si l'on prend en compte la rareté des meubles de ce blason et l'originalité de sa composition, ces différences peuvent être négligées. Le patronyme associé à ce blason est Simonnet; sur l'un des recueils de l'Armorial de Paris ${ }^{5}$, le blason est celui d'un agent de change.

\section{Interprétation}

11 La famille représentée (cf. fig. 2) appartient à la paroisse Saint-Eusèbe d'Auxerre à une époque que le style du vitrail permet de situer entre le troisième quart du $\mathrm{XvI}^{\mathrm{e}}$ siècle et le premier quart du xvII ${ }^{\mathrm{e}}$ siècle.

12 Pour l'identifier, nous avons exploité les registres paroissiaux de cette paroisse et cherché à savoir si le patronyme Simonnet (Symonet) était représenté à Auxerre et, plus précisément, dans cette paroisse, à l'époque considérée. Dans un second temps, il fallait espérer trouver la trace d'un mariage, dont l'épouse porterait le patronyme Symonet.

13 Les registres paroissiaux d'Auxerre, et singulièrement ceux de la paroisse Saint-Eusèbe, sont extrêmement lacunaires. Aucun acte n'est répertorié pour Saint-Eusèbe entre 1564 et 1580 , ni entre 1590 et 1595 . Les actes de mariage ainsi que les actes de décès sont totalement absents, les premiers mariages n'apparaissant qu'en 1640. Une exploitation systématique des actes de naissance a cependant apporté un résultat intéressant. Parmi les paroissiens figure un couple dans lequel l'épouse est Germaine Simonnet, nom le plus souvent orthographié Symonet. Elle est mariée à Charles Rousselet et le tableau cidessous donne la liste des enfants du couple Charles Rousselet et Germaine Symonet, entre 1596 et 1619 - mais il ne faut pas oublier que 1596 est la première année qui suit une lacune de cinq ans, et donc la première naissance relevée peut ne pas être la première de ce couple.

\begin{tabular}{|l|l|l|l|}
\hline Date de naissance & Identité & Mariage & Signe encore le \\
\hline 23011596 & Rousselet Perrette & 15021627 & 091644 \\
\hline 12091598 & Rousselet Jean & & \\
\hline 19091600 & Rousselet Charles & & \\
\hline 20021602 & Rousselet Nicolas & 28111618 & \\
\hline 09011604 & Rousselet Claude (fille) & & \\
\hline 06021605 & Rousselet Jean & & 021634 \\
\hline 27021607 & Rousselet Germaine & 22111633 & 111643 \\
\hline 09021610 & Rousselet Marie & 27011634 & 111643 \\
\hline 22061612 & Rousselet Anne & & \\
\hline
\end{tabular}




\begin{tabular}{|l|l|l|l|}
\hline 12071614 & Rousselet Charles & & 091629 \\
\hline 13101616 & Rousselet Jean & 13011670 & \\
\hline 13121619 & Rousselet Chrétienne & Ursuline (31 10 1633) & $\dagger$ mars 1646 \\
\hline
\end{tabular}

14 En l'absence, à Auxerre, d'un autre couple dont l'épouse porterait ce patronyme, notre conclusion est que la famille représentée sur le vitrail est bien celle de Charles Rousselet et Germaine Symonet. La date de leur mariage n'est pas connue et aucun contrat de mariage n'a été retrouvé. Le tableau ci-dessus montre la naissance, entre 1596 et 1619, de six fils : sur le vitrail de l'église de Saint-Eusèbe (cf. fig. 2) quatre jeunes hommes accompagnent le père de famille et deux "enfants emmaillotés", placés derrière la cohorte des vivants, reprenant un meuble du blason attribué à la famille Symonet, pourraient représenter le souvenir de deux enfants mâles décédés avant la réalisation de la verrière.

15 Un certain nombre d'éléments nous permettent de proposer qu'il s'agît de familles aisées, composées d'hommes de loi et de gens de finances.

\section{La famille Symonet}

Germaine Symonet signe un certain nombre d'actes de baptême en tant que marraine. Sa signature est fort belle, ce qui suppose un bon niveau d'éducation. Elle signe, en juin $1590^{6}$, un acte de baptême où elle est qualifiée fille de Drouet Symonet. Sa signature sur un acte de baptême de mars 1598 est représentée ci-dessous ${ }^{7}$ (fig. 4).

Fig. 4 - Signature de Germaine Symonet sur un acte de baptême, registre de la paroisse SaintEusèbe daté de 1598

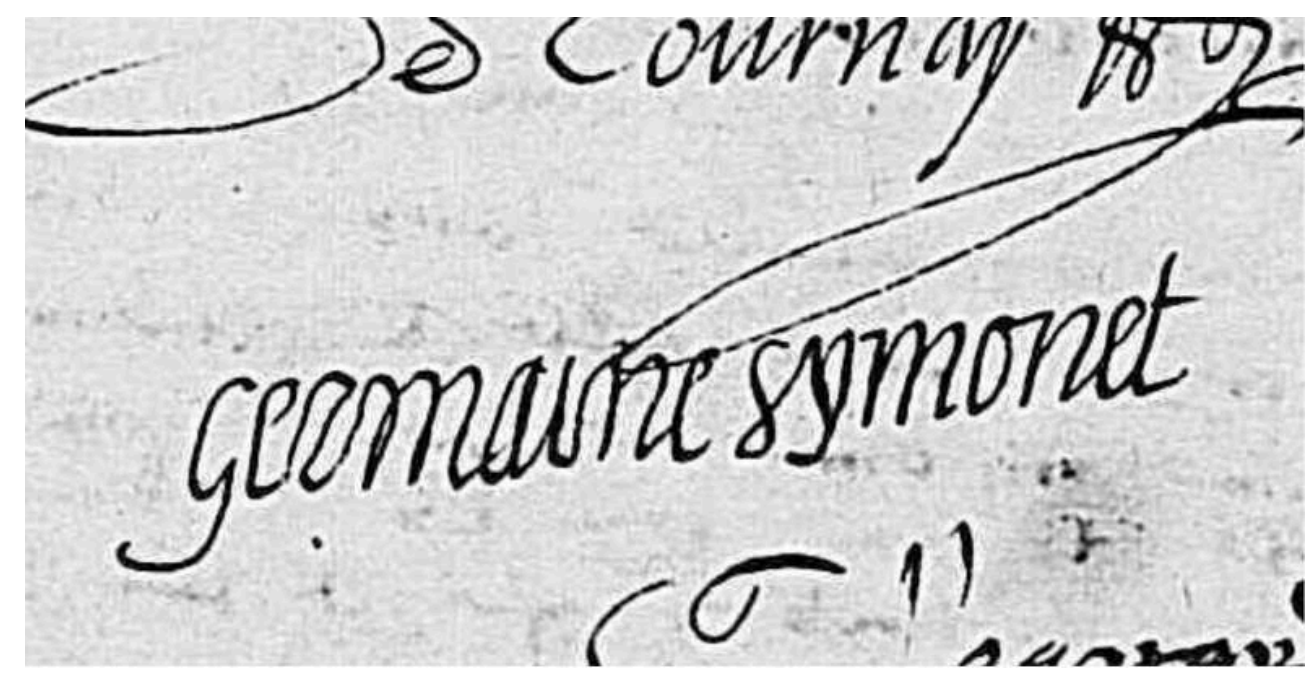

Archives dép. Yonne 4E 24 E 22

17 Elle est alors désignée épouse de Charles Rousselet. La date de son mariage se situe donc entre 1590 et 1595, le baptême de leur premier enfant connu se situant en janvier 1596. 
Son père Drouet Symonet était receveur ordinaire du domaine du roi en son comté d'Auxerre. Il avait épousé le 15 janvier 1543 Marie Cochon. De nombreux membres de cette famille occupaient des postes d'avocat ou de procureur dans la ville d'Auxerre. Un certain Claude apparaît souvent sur les registres paroissiaux et signe en tant que parrain l'acte de baptême de Jean Rousselet, fils de Charles et Germaine Symonet, en 1598. Il est alors procureur du roi en l'élection d'Auxerre. Il avait un frère, Hélie, à Paris, procureur au Parlement de Paris dont l'inventaire après décès, daté de 1594 et signé par Claude, est conservé aux Archives nationales ${ }^{8}$.

\section{La famille Rousselet}

19 La présence de Charles Rousselet dans les documents d'archives est plus discrète. Présenté comme fils de Jean, il signe en janvier 1585 comme parrain un acte de baptême à l'église Saint-Eusèbe ${ }^{9}$. Le 24 septembre 1590, il apparaît sur un autre acte de baptême (fig. 5) célébré à Saint-Pierre-en-Vallée ${ }^{10}$, toujours présenté comme fils de Jean Rousselet.

Fig. 5 - Signature de Charles Rousselet sur un acte de baptême, registre de la paroisse de SaintPierre-en-Vallée, daté de 1590

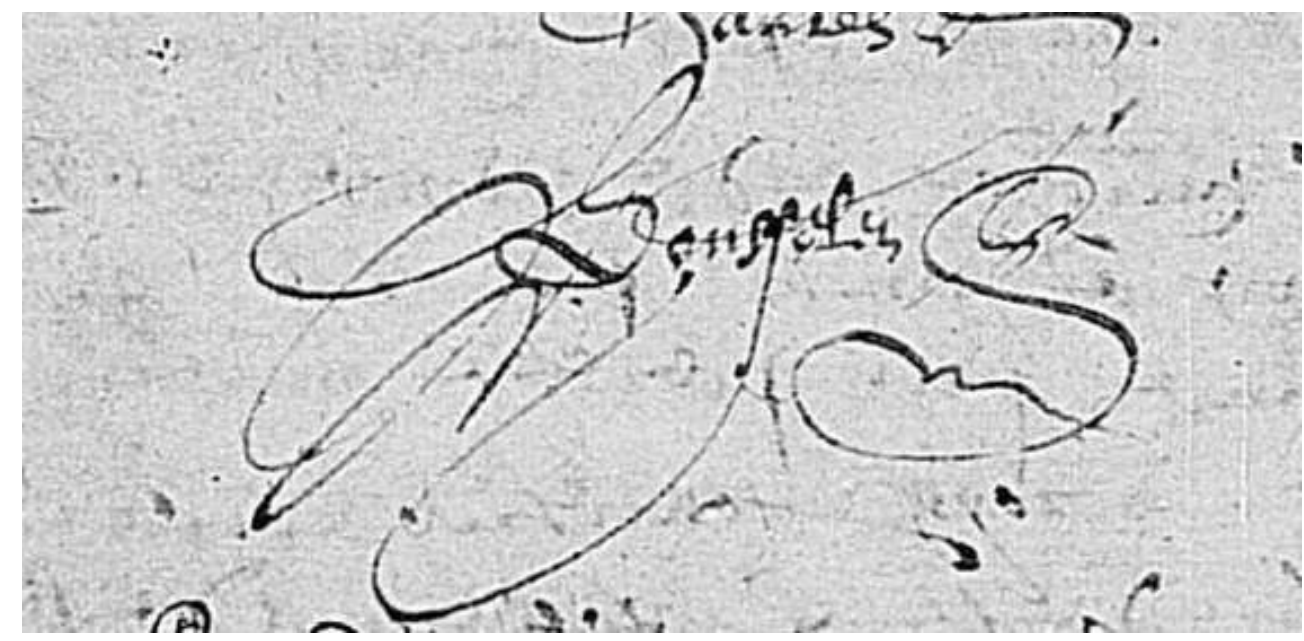

Archives dép. Yonne 4E 24 E 24

Jean Rousselet, son père, est donné comme marchand, puis comme "grainetier". De 1587 à 1593, soit avant la naissance de son premier enfant et sans doute de son mariage, Charles a été commis par le trésorier général de France en Bourgogne pour rendre les comptes de feu Drouet Symonet, père de sa future épouse. En 1600, il est dit receveur des tailles. En 1615, sa profession est donnée comme « grainetier au grenier à sel et magasin de la ville d'Auxerre ».Un examen du fonds de la paroisse Saint-Eusèbe montre à deux reprises, en 1610 et $1641^{11}$, la participation de Charles Rousselet à des activités financières liées à la fabrique de l'église.

En 1632, le jour de la Saint-Michel, il signe le registre paroissial de Saint-Eusèbe, choisi comme parrain de son petit-fils, Charles Bibault, fils de sa fille Perrette ${ }^{12}$ (fig. 6). 
Fig. 6 - Signature de Charles Rousselet sur l'acte de baptême de son petit-fils Charles Bibault, registre de la paroisse Saint-Eusèbe, daté de 1632

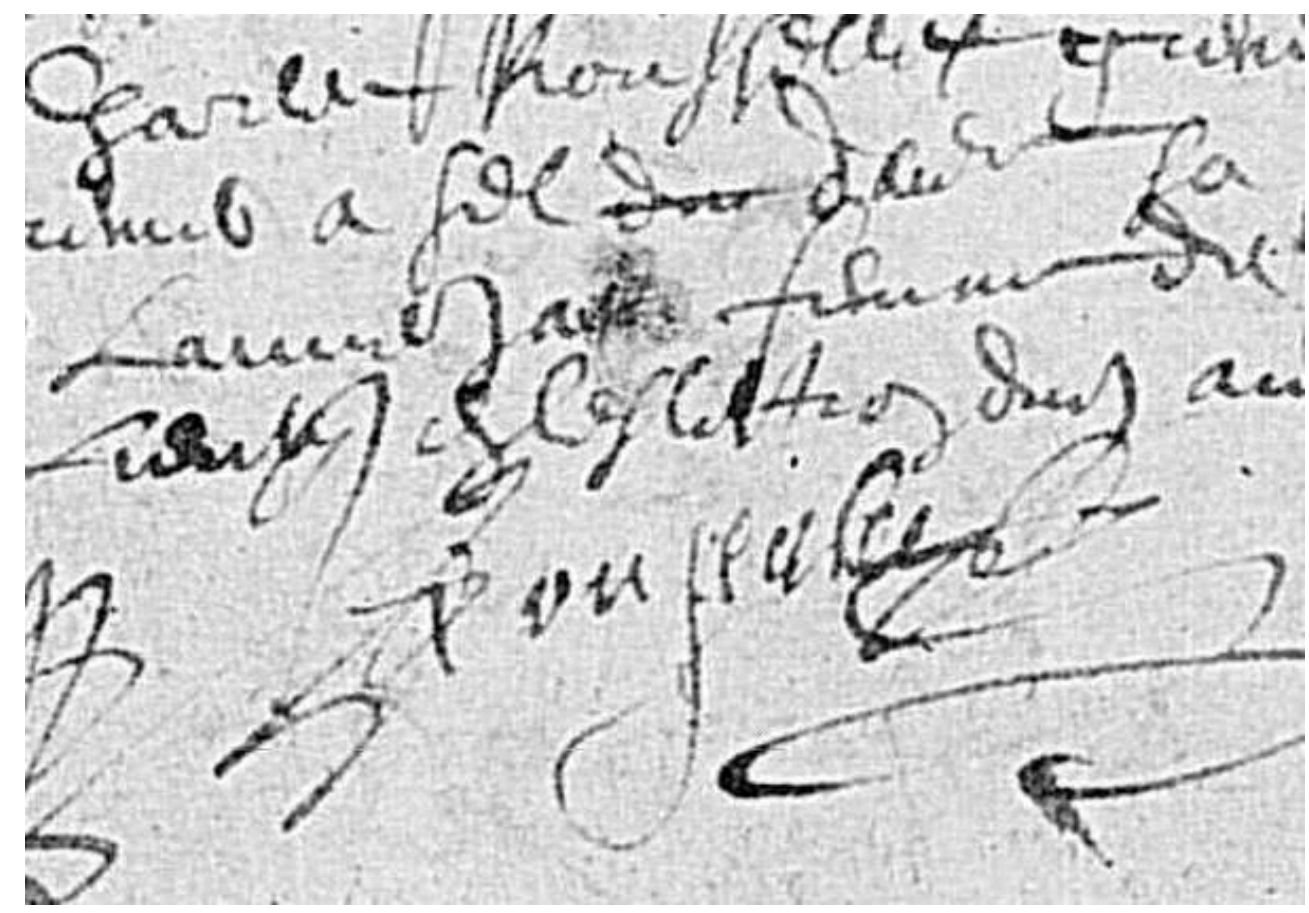

Archives dép. Yonne 4E 24 E 86

Son épouse, Germaine Symonet, est qualifiée de veuve sur un acte de mariage daté de 1644 , acte signé par plusieurs de ses enfants. Un document de la fabrique faisant état de sa présence en 1641, la date du décès de Charles Rousselet se situe donc entre 1641 et 1644.Comme déjà mentionné pour la famille Symonet, la famille Rousselet compte des membres à Paris. Sans une étude précise et détaillée, il reste impossible de relier entre eux les Rousselet de Paris avec ceux d'Auxerre. Si l'analyse des faits présentés cidessus est correcte, alors le blason 1 (à gauche sur la fig. 1) correspondrait à celui de la famille Rousselet d'Auxerre. Mais la démonstration s'arrête là.

Il importe cependant de citer l'érudit abbé Lebeuf (1687-1760) : « Monsieur Chacheré de la Brosse, qui est venu d'une Rousselet, dont le père était sieur de la Brosse, ne sçait pas quelles étaient ses armoiries. Elles ne sont ni à la Brosse, ni à Auxerre dans leur ancienne maison ${ }^{13}$.» Or, le 11 août 1681, Anne Rousselet épousait, à Saint-Eusèbe, Pierre Chacheré et accomplissait la fusion des Rousselet dans les Chacheré. Ce blason inconnu a donc peu de chance d'être trouvé et documenté dans notre siècle... hors de l'église Saint-Eusèbe.

\section{La famille Vincent}

Le dernier blason, sur le prie-Dieu du père de famille, associe les armes définies cidessus comme celle des Rousselet, à dextre, à d'autres armes non répertoriées (fig. 7). 
Fig. 7 - Blason de Charles Rousselet, posé sur son prie-Dieu, associant les armes de son père, Jean, à dextre, et de sa mère, Perrette Vincent, à sénestre

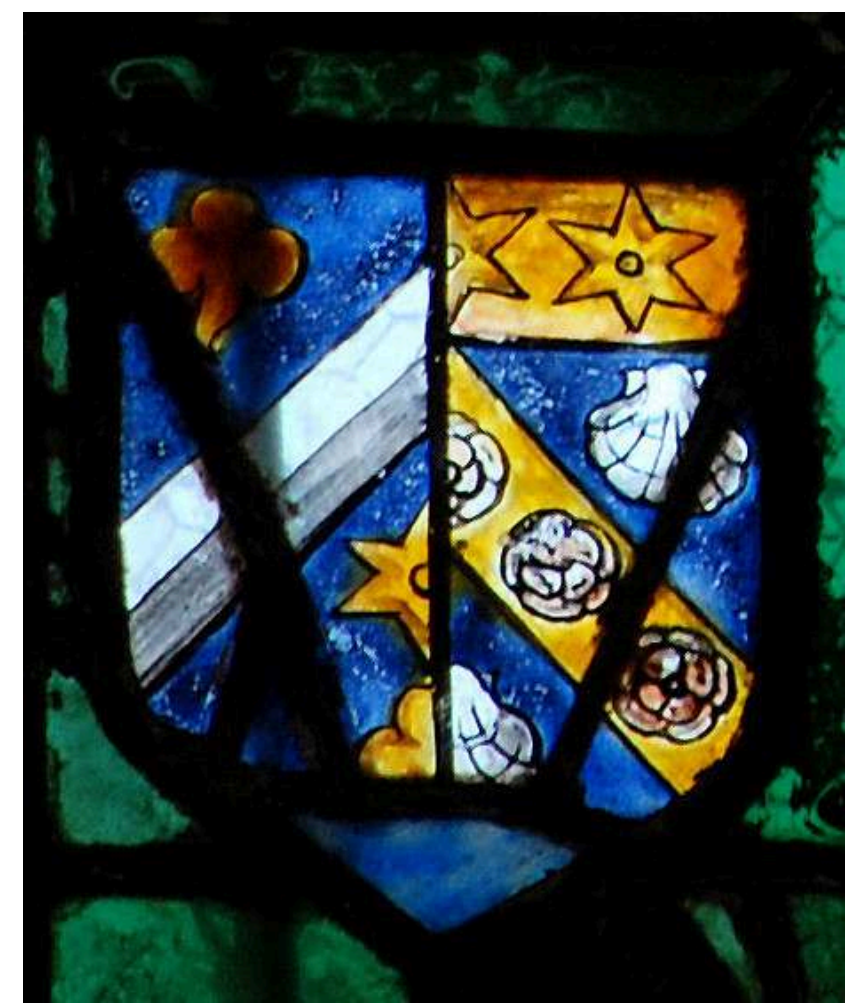

Cl. P. Wahlen celles de son père. Un document, conservé parmi les archives notariales aux Archives départementales de l'Yonne, est la minute d'un acte du 2 juin 1599 passé entre Charles Rousselet et Jean Rousselet, son père ${ }^{14}$, qui transigent à propos de l'héritage de Perrette Vincent, la mère de Charles, qui avait été, en premières noces, l'épouse de Claude Charles. Le testament de Perrette figure parmi les minutes de $\mathrm{M}^{\mathrm{e}}$ Pierre Fauleau à la date du 18 mai $1584^{15}$. Elle mentionne plusieurs enfants issus de ses deux mariages, dont Charles Rousselet. Ce document nous permet donc d'évoquer la famille maternelle de Charles Rousselet. D'après un document sur la famille Vincent ${ }^{16}$, Perrette était la sœur d'Edme Vincent qui a occupé successivement, entre 1559 et 1585, les positions de lieutenant de la prévôté d'Auxerre, avocat au baillage, bailli de l'évêque et était seigneur du Petit Monéteau. Fils unique, il compte parmi ses enfants et petits-enfants des avocats, contrôleurs au grenier à sel et autres lieutenants au baillage.

\section{Conclusion}

Les membres des familles Symonet, Rousselet et Vincent étaient des hommes de loi et de finance à Auxerre, mais aussi à Paris. Un tableau rassemble la liste des enfants du couple Charles Rousselet et Germaine Symonet dont nous avons retrouvé la trace : six fils et six filles, qui ont reçu le sacrement de baptême à Saint-Eusèbe entre 1596 et 1619 . Quatre filles se sont mariées. Chrestienne, la dernière-née, est entrée en religion en 1633 au couvent des Ursulines, voisin de l'église Saint-Eusèbe ${ }^{17}$, et décédée dans ce monastère le 9 mars $1646^{18}$. On trouve au fil des registres paroissiaux mention (avec

Bulletin du centre d'études médiévales d'Auxerre | BUCEMA, 20.2 | 2016 
signatures) de Jean et Charles Rousselet, enfants du couple Rousselet-Symonet. Mais aucune trace de Nicolas, qui a pu mourir en bas âge. Jean s'est marié à l'âge de cinquante-trois ans. Les registres des autres paroisses de la ville d'Auxerre ne montrent aucune famille, à cette période, dont l'épouse porterait le patronyme Symonet. La verrière a dû être commandée par un paroissien aisé de Saint-Eusèbe, proche de la fabrique, dont l'épouse portait ce patronyme. Cette commande est probablement intervenue après la naissance des enfants, soit après 1619, mais avant le décès de Charles Rousselet survenu après juin 1641 et avant septembre 1644. Le marché pour cette verrière n'a pas été conservé, mais $\mathrm{H}$. Forestier cite, parmi les minutes $\left(\mathrm{M}^{\mathrm{e}}\right.$ Pierre Leclerc) qu'il a dépouillées, un marché passé le 14 mars 1578 entre Claude Rousselet, enquêteur au siège du baillage d'Auxerre, et Nicolas Hure, peintre à Auxerre, qui s'engage à faire à Notre-Dame-la-d'Hors vis-à-vis du grand autel une verrière où seront représentés « un crucyfyment, les ymaiges Nostre Dame, Sainct Jean, la Magdelaine, Sainct Germain, Sainct Claude, les effigies dudit Rousselet, de trois fils masles et quatre filles (...) habillés de habitz convenables, avec les armoiries dudit Rousselet et sa femme et encores faire ung ymaige de Dieu le père par-dessus les ymaiges dessusd" moyennant vingt écus ${ }^{19}$. Cette verrière de 1578 , bien antérieure à celle donnée par Charles Rousselet et Germaine Symonet à Saint-Eusèbe, n'est pas conservée et nous ne connaissons pas le détail des liens familiaux entre Claude et Charles Rousselet. Mais cet exemple montre bien que, dans cette société auxerroise, la commande de verrières était, dans le milieu que nous avons évoqué, une démarche traditionnelle. Il nous reste à Saint-Eusèbe les visages de Charles Rousselet et quatre de ses fils en habits convenables; ses armoiries, celles de sa femme Germaine Symonet et de sa mère Perrette Vincent. L'autre partie de la verrière, symétrique de celle-ci, où priaient son épouse et ses filles, a disparu.

Reçu : 6 octobre 2016 - Accepté : 6 décembre 2016

\section{NOTES}

1. "Retour sur le vitrail à Auxerre à la fin du $\mathrm{xvI}^{\mathrm{e}}$ siècle ", Mémoires de l'Académie des sciences, arts et belles-lettres de Dijon, 147 (2011-2012), p. 303-310 (avec bibliographie).

2. Voir à Charenton-le-Pont, Médiathèque du Patrimoine, les dossiers de restauration ou d'entretien depuis 1848 (série 0081/089).

3. F. PERROT, « Retour sur le vitrail... », op. cit., p. 309.

4. D’HozIER, Armorial général de France, 1696, Paris, t. 2 [PARIS, BnF, fr. 32251], p. 1497.

5. D’HOzIER, Armorial général de France, 1696, Paris, t. 1 [PARIS, BnF, fr. 32250], p. 483.

6. Archives départementales de l'Yonne [désormais ADY], registre de N.D. de la Hors, 5/Mi 93/3, vue 330 .

7. ADY, registre de la paroisse Saint-Eusèbe, 5/Mi 97/5, vue 203.

8. Paris, Archives nationales, Minutier Central/ET/XXIII/135.

9. ADY, 5 Mi 98/1 (1581-1667), vue 91.

10. ADY, 5 Mi 108/2 (1582-1611), vue 197.

11. ADY, G 2367. 
12. ADY, 5 Mi 97/6 (1617-1633), vue 300.

13. J. LEBEUf, Lettres, éditées par MM Quantin et Cherest, t. 1, Auxerre, 1866, p. 403.

14. $\mathrm{ADY}, 3 \mathrm{E} 7 / 107$, pièce 46.

15. $\mathrm{ADY}, 3 \mathrm{E} 7 / 170$, pièce 30 .

16. Document inédit de la Société généalogique de l'Yonne.

17. ADY, 3 E 6/368, 31 octobre 1633.

18. ADY, 5 Mi 115/2, vue 6.

19. H. FORESTIER, Répertoire et inventaires de fonds déposés par les notaires de l'Yonne. Études de $M^{e}$ Rolland Sampic, notaire à Seignelay et de $M^{e}$ Louis Jouvin, notaire à Auxerre, Auxerre, 1942, p. 226.

INDEX

Mots-clés : Auxerre, église Saint-Eusèbe, XVIe-XVIIe siècles, héraldique, vitrail, familles auxerroises (Symonet, Rousselet, Vincent)

\section{AUTEURS}

DANIELLE VELDE

Chercheur indépendant

\section{FRANÇOISE PERROT}

Directeur de recherche honoraire, CNRS/Laboratoire de médiévistique occidentale de Paris (LAMOP) 